\title{
Benign Anal Neoplasm
}

National Cancer Institute

\section{Source}

National Cancer Institute. Benign Anal Neoplasm. NCI Thesaurus. Code C4611.

A non-metastasizing neoplasm arising from the anus. Representative examples include squamous papilloma and papillary hidradenoma. 